\title{
AVALIAÇÃO DA TERAPIA FOTODINÂMICA COM AZUL DE METILENO EM Leishmania major e Leishmania braziliensis: ESTUDO in vitro
}

\author{
Danielle El Atra Coelho' \\ Letícia Corrêa Fontana² \\ Juliana Guerra Pinto ${ }^{3}$ \\ Juliana Ferreira-Strixino ${ }^{4}$
}

Resumo: A Leishmaníase é uma doença crônica causada pelo protozoário do gênero Leishmania, cujo tratamento é agressivo. A Terapia Fotodinâmica (TFD) é uma alternativa promissora que combina luz, fotossensibilizador (FS) e oxigênio molecular, para causar a morte celular. O objetivo desse trabalho foi avaliar, in vitro, a ação da TFD com Azul de metileno (AM) em promastigotas de Leishmania, por teste de MTT, curva de crescimento e morfologia do parasito. O teste de MTT demonstrou alteração de ambas as espécies após interação com o AM no escuro e após TFD. A análise das curvas demonstrou que a TFD influenciou o crescimento das espécies. A análise morfológica revelou que o AM no escuro não causou alterações expressivas como a TFD, sendo a cepa de L. braziliensis mais afetada que a cepa de L. major. Pode-se concluir que a TFD com AM foi promissora contra promastigotas de Leishmania, pois foi capaz de diminuir o crescimento e alterar a morfologia dos parasitos em cultura.

Palavras-chave: Terapia fotodinâmica; Leishmaniose tegumentar americana; Azul de metileno.

\footnotetext{
1 Laboratório de Terapia Fotodinâmica/Instituto de Pesquisa e Desenvolvimento/Universidade do Vale do Paraíba- UNIVAP), Laboratório de Terapia Fotodinâmica, Brasil. E-mail: danielle.eacoelho@gmail.com.

2 Laboratório de Terapia Fotodinâmica/Instituto de Pesquisa e Desenvolvimento/Universidade do Vale do Paraíba- UNIVAP), Laboratório de Terapia Fotodinâmica, Brasil. E-mail: leticia.fontana@yahoo.com.br.

3 Laboratório de Terapia Fotodinâmica/Instituto de Pesquisa e Desenvolvimento/Universidade do Vale do Paraíba- UNIVAP), Laboratório de Terapia Fotodinâmica, Brasil. E-mail: jgbiomd@gmail.com.

4 Laboratório de Terapia Fotodinâmica/Instituto de Pesquisa e Desenvolvimento/Universidade do Vale do Paraíba- UNIVAP), Laboratório de Terapia Fotodinâmica, Brasil. E-mail: juferreira@univap.br.
} 